\title{
Effects of regional anesthesia techniques on local anesthetic plasma levels and complications in carotid surgery: a randomized controlled pilot trial
}

Thomas Rössel ${ }^{1 *+} \mathbb{D}$, Christopher Uhlig ${ }^{1 \dagger}$, Jörg Pietsch², Stefan Ludwig ${ }^{3}$, Thea Koch¹, Torsten Richter ${ }^{1}$, Peter Markus Spieth ${ }^{1}$ and Stephan Kersting ${ }^{3,4}$

\begin{abstract}
Background: The ultrasound guided intermediate cervical plexus block with perivascular infiltration of the internal carotid artery (PVB) is a new technique for regional anesthesia in carotid endarterectomy (CEA). We conducted a pilot study investigating the effects of deep cervical block (DCB), intermediate cervical block alone (ICB) and PVB on perioperative complications in patients undergoing elective CEA. We hypothesized, that the ropivacaine plasma concentration is higher in patients receiving DCB compared to PVB and ICB.
\end{abstract}

Methods: In a randomized controlled pilot study thirty patients scheduled for elective CEA were randomly assigned into three groups: DCB receiving $20 \mathrm{~mL}$ ropivacaine $0.5 \%(n=10)$, ICB receiving $20 \mathrm{~mL}$ ropivacaine $0.5 \%(n=10)$ and PVB receiving $20 \mathrm{~mL}$ ropivacaine $0.5 \%$ and $10 \mathrm{~mL}$ ropivacaine $0,3 \%(n=10)$. As primary outcome, plasma levels of ropivacaine were measured with high performance liquid chromatography before, 5, 10, 20,60, and $180 \mathrm{~min}$ after the injection of ropivacaine. Secondary outcomes were vascular and neurological complications as well as patients' and surgeons' satisfaction. All analyses were performed on an intention-to-treat basis. Statistical significance was accepted at $p<0.05$.

Results: No conversion to general anesthesia was necessary and we observed no signs of local anesthetic intoxication or accidental vascular puncture. Plasma concentration of ropivacaine was significantly higher in the DCB group compared to PVB and ICB $(p<0.001)$ and in the PVB group compared to ICB $(p=0.008)$. Surgeons' satisfaction was higher in the PVB group compared to ICB $(p=0.003)$ and patients' satisfaction was higher in the PVB group compared to ICB ( $p=0.010)$ and DCB group ( $p=0.029)$. Phrenic nerve paralysis was observed frequently in the DCB group $(p<$ 0.05). None of these patients with hemi-diaphragmatic paralysis showed signs of respiratory distress.

Conclusion: The ultrasound guided PVB is a safe and effective technique for CEA which is associated with lower plasma levels of local anesthetic than the standard DCB. Considering the low rate of complications in all types of regional anesthesia for CEA, larger randomized controlled trials are warranted to assess potential side effects among the blocks.

\footnotetext{
*Correspondence: thomas.roessel@ukdd.de

†Thomas Rössel and Christopher Uhlig contributed equally to this work.

'Department of Anaesthesiology and Critical Care Medicine, University

Hospital Carl Gustav Carus Dresden, Technische Universität Dresden,

Fetscherstr. 74, 01307 Dresden, Germany

Full list of author information is available at the end of the article
}

(c) The Author(s). 2019 Open Access This article is distributed under the terms of the Creative Commons Attribution 4.0 International License (http://creativecommons.org/licenses/by/4.0/), which permits unrestricted use, distribution, and reproduction in any medium, provided you give appropriate credit to the original author(s) and the source, provide a link to the Creative Commons license, and indicate if changes were made. The Creative Commons Public Domain Dedication waiver (http://creativecommons.org/publicdomain/zero/1.0/) applies to the data made available in this article, unless otherwise stated. 
(Continued from previous page)

Trial registration: The trial was registered at German Clinical Trials Register (DRKS) on 04/05/2019 (DRKS00016705, retrospectively registered).

Keywords: Carotid endarterectomy, Cervical plexus block, Plasma concentration, Regional anesthesia, Local anesthetic, Ropivacaine

\section{Background}

In carotid endarterectomy (CEA), regional anesthesia is associated with beneficial effects regarding sensitivity and specificity of patients neurological monitoring $[1,2]$. CEA in awake patients requires the blockade of cervical nerves from $\mathrm{C} 2$ to $\mathrm{C} 4$. The blockade can be performed on the nerve roots or on the terminal nerve fibers. The most frequently used regional anesthetic techniques for this purpose are superficial, intermediate and deep cervical block. The anesthetic effects of these three techniques are comparable $[3,4]$. However, during dissection of the internal carotid artery (ICA) the need for local anesthetic supplementation by the surgeon ranges from 20 to $60 \%$ [5].

Over the last decade, the use of ultrasound has improved the safety and efficacy of regional anesthesia $[6,7]$. The major advantages of ultrasound-guided regional anesthesia are the visualization of the target structures, the direct observation of the spread of the local anesthetic and the reduction of puncture-related complications compared to nerve stimulation or land mark technique. Furthermore, new ultrasound-guided anesthetic approaches for blockade of various nerves were developed [8-11]. Our group previously demonstrated a good clinical efficacy with a low rate of intraoperative local anesthetic supplementation by surgeons for the combination of ultrasound-guided intermediate cervical block with perivascular infiltration of the ICA, so called perivascular block (PVB) [8]. On the other hand, due to the vicinity of the vessels the PVB may result on higher plasma levels of local anesthetic compared to ultrasoundguided intermediate cervical block alone (ICB). This may cause more perioperative complications such as dizziness and seizures as described for the deep cervical block (DCB) [4]. In addition to potential toxic effects of local anesthetics, respiratory distress by phrenic nerve paralysis is possible [4].

To our knowledge, ultrasound-guided PVB, ICB and DCB have not been assessed in regard to block performance, perioperative complications and plasma levels of local anesthetics.

Therefore, we investigated the effects of PVB, ICB and DCB on ropivacaine plasma levels, anesthesia related nerve paralysis and efficacy of the block in patients undergoing elective CEA. We hypothesized that the ropivacaine plasma concentration is higher in patients receiving DCB for elective CEA compared to PVB and ICB.

\section{Methods}

We conducted a randomized controlled, single-center pilot study. The trial is reported according to the Consolidated Standards of Reporting Trials (CONSORT) statement [12]. The experimental protocol is depicted in Fig. 1. After approval by the local institutional review board of the Technische Universität Dresden, Germany (EK 130042013), thirty consecutive patients scheduled for elective CEA in the University Hospital Carl Gustav Carus, Dresden, Germany were screened for eligibility in a 6 month period. Inclusion and exclusion criteria are summarized in Table 1 . The patients were randomized directly before start of ultrasound-guided regional anesthesia to three groups: DCB with $20 \mathrm{~mL}$ ropivacaine $0.5 \%$, ICB alone with $20 \mathrm{~mL}$ ropivacaine $0.5 \%$ and combination of intermediate cervical block and perivascular infiltration, PVB, with $20 \mathrm{~mL}$ ropivacaine $0.5 \%$ and 10 $\mathrm{mL}$ ropivacaine $0.3 \%$, respectively. The random sequence was compiled using a computer-generated random numbers table and group allocation was concealed by sequentially numbered opaque closed envelopes. Surgeons and data collectors were blinded to the study group.

\section{Regional anesthesia}

Regional anesthesia was performed by two senior anesthesiologists with substantial experience in performance of ultrasound guided deep and intermediate cervical plexus block. The regional anesthetic techniques used in this study were performed as previously described $[6,8]$. Briefly, patients were placed for regional anesthesia in supine position with their heads turned $30^{\circ}$ to the opposite side. Prior to performing the block, the anatomic conditions of the neck region were analyzed by ultrasound. During this examination, first the transverse process with the corresponding nerve roots from the second to the seventh cervical vertebrae $(\mathrm{C} 2$ to $\mathrm{C} 7)$ as well as the distal part of cervical plexus were visualized and recorded using a Philips HD 11 with a $12.5 \mathrm{MHz}$ linear ultrasound transducer (Philips Medicine Systems $\mathrm{GmbH}$, Hamburg, Germany). Subsequently, the ICA was identified and the distance between the skin and the ICA was recorded. The cervical block was performed according to group allocation (Fig. 2). The success of the 


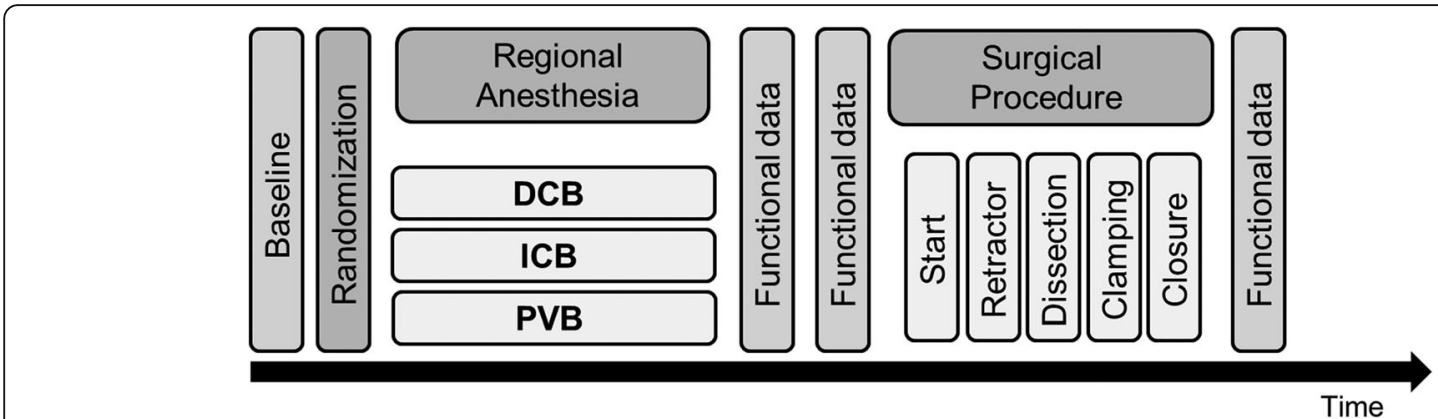

Fig. 1 Time course of intervention. DCB: deep cervical block, ICB: intermediate cervical block, PVB: intermediate cervical block with perivascular infiltration of the internal carotid artery

blockade was evaluated 5,10 , and $15 \mathrm{~min}$ after regional anesthesia by pin prick test in the dermatomes from $\mathrm{C} 2$ to C5. Additionally, $20 \mathrm{~min}$ after the block was performed, sensory skin testing at the hand, shoulder and motor testing at the wrist, arm and shoulder were performed. Puncture related complications, such as respiratory distress, hypoglossal and facial nerve palsy or Horner's syndrome were also assessed.

\section{Intraoperative management and hemodynamic monitoring}

The evening before surgery, patients received $25 \mathrm{mg}$ of clorazepate (Aventis $\mathrm{GmbH}$, Bernburg, Germany) per os by request. No premedication was administered at the day of surgery. In the operating room, peripheral venous access, a 5-lead ECG including ST-segment analysis, a pulsoxymetry and an arterial line for continuous monitoring of arterial blood pressure were placed. Hemodynamic data were continuously recorded using a Philips Intellivue MP 70 (Philips Medicine Systems GmbH, Hamburg, Germany). An arterial blood gas analysis was performed before, as well as 15 and $30 \mathrm{~min}$ after regional anesthesia. To improve intraoperative comfort, all patients received $0.03 \mu \mathrm{g} / \mathrm{kg} / \mathrm{min}$ remifentanil (Aspen-Germany GmbH, Germany; dosage in relation to ideal body weight). After surgery, the patients were observed for $24 \mathrm{~h}$ under cardiovascular and neurological monitoring in the intermediate care unit, post anesthesia care unit or regular ward, as appropriate.

\section{Surgical management}

All CEAs were performed by two senior vascular surgeons. Surgery was started when the surgical site had been sufficiently anesthetized. Pain was intraoperatively evaluated by means of the Numeric Analgesia Scale (NAS) graded from 0 (no pain) to 10 (worst pain) during the performance of regional anesthesia and during skin incision, retractor placement, dissection, cross-clamping, and skin closure. If patients complained of intraoperative pain NAS $>2$ an additional local infiltration of lidocaine 1\% (mibe GmbH, Brehna, Germany) was administered by the surgeon in $1 \mathrm{ml}$ steps until a sufficient anesthetic level was achieved. The total amount of supplemented lidocaine was recorded. At the end of surgery, the surgeons assessed the surgical conditions on a subjective scale ranging from 1 to 5 (1-very good, 2-good, 3reasonable, 4-poor, 5 -very poor). All patients underwent follow-up visits on the first post-operative day. Patients were asked to assess their satisfaction with anesthesia in five grades from 1-very good, 2-good, 3-reasonable, 4poor to 5 -very poor and if they would again undergo surgery under regional anesthesia.

\section{Plasma level measurement}

Arterial blood samples for plasma level of ropivacaine were collected before and 5, 10, 20, 60, and $180 \mathrm{~min}$ after the injection of ropivacaine. After immediate centrifugation, plasma samples were stored at $-20^{\circ} \mathrm{C}$. The unbound ropivacaine plasma level was measured by the Institute of Legal Medicine, Dresden Technical University. After a liquid-liquid extraction procedure for sample preparation specimens were analyzed with a high performance liquid chromatography photodiode array detection system (Agilent 1100 series, Agilent Technologies, Waldbronn, Germany). For quantification, drugfree serum was spiked at five different concentrations of ropivacaine $(100,200,500,1000,2000 \mathrm{ng} / \mathrm{ml})$. Ropivacaine concentration was calculated using linear

Table 1 Inclusion and exclusion criteria. CEA: carotid endarterectomy, ICA: internal carotid artery

\begin{tabular}{ll}
\hline Inclusion & Exclusion \\
\hline -age $>18$ years & -history of anaphylactic reaction to local anesthetics \\
-elective CEA surgery for treatment of ICA stenosis & -local infection in the lateral cervical region \\
-written informed consent & -presumed limitation of patients' compliance \\
\hline
\end{tabular}





Fig. 2 Ultrasound images of cervical block. a: deep cervical block, b: intermediate cervical block, c: intermediate cervical block with perivascular infiltration of the internal carotid artery. Ultrasound images were acquired with Philips HD-11-XE (Philips Healthcare GmbH, Hamburg, Germany using a linear probe (12 MHz, L-12-4, Philips Healthcare GmbH, Hamburg, Germany). Direction of the ultrasound enhanced puncture needle is depicted as green dashed line and the needle tip as green cross. Yellow line: superficial cervical fascia, blue line: deep cervical fascia. LA: local anesthetic, ECA: external carotid artery, ICA: internal carotid artery, IJV: internal jugular vein, CCA: commune carotid artery, TP: Processus transversus of the respective cervical vertebra, V: ventral, D: dorsal, SM: M. steroncleidomastoideus. *: C5 nerve root, **: C6 nerve root, a: N. vagus, x: N. auricularis magnus and N. transversus colli

regression. The limit of quantification of the method was $100 \mathrm{ng} / \mathrm{ml}$.

\section{Assessment of phrenic nerve paresis}

The quantitative analysis of phrenic nerve paresis was performed by electrical impedance tomography (EIT, PulmoVista 500, Dräger Medical, Lübeck, Germany) [13]. Images were obtained at baseline, 15, 30 and 180 min after successful establishment of the cervical block. The images containing $32 \times 32$ pixels were recorded at a rate of 50 frames/s during $2 \mathrm{~min}$ for offline analysis. Using a MATLAB (Vers. R2006b, The Mathworks Inc., Natick, MA, USA) based routine, changes in impedance (region of interest - ROI) were determined. The ROI was divided into two zones with equal size corresponding with the left and right lung. Relative changes in impedance were computed. A phrenic nerve paresis was defined as a decrease in change of impedance of more than $50 \%$ compared to baseline in one ROI. Phrenic nerve paresis was assessed qualitative and quantitatively by an investigator blinded to the group allocation.

\section{Neurological monitoring}

Neurologic function was perioperatively continuously monitored by observing the level of consciousness and the response to verbal commands. During $5 \mathrm{~min}$ test cross-clamping of the ICA, the patient was challenged to squeeze a squeaking rubber toy with the contralateral hand every $10-15 \mathrm{~s}$ and to answer simple questions for close judgment of neurological function. A shunt was placed if any signs of neurological dysfunction occurred during test cross-clamping. Additionally, the function of recurrent laryngeal nerve, hypoglossal nerve and facial nerve was monitored before and $30 \mathrm{~min}$ after regional anesthetic blockade, as well as before and after cross clamping and at the end of surgery.

\section{Statistical analysis}

Statistical analysis was performed with SPSS (Vers. 20, IBM Deutschland GmbH, Ehningen, Germany). Graphs were computed using Graph Pad Prism Vers. 6.01 (GraphPad Software Inc., La Jolla, CA, USA). Values are given as total numbers and percentage, mean and standard deviation or median and interquartile range as appropriate. Statistical significance was considered at twosided $p<0.05$. All analyses were performed on an intention-to-treat basis. Normal distribution was assessed visually using Q-Q Plot of standardized residuals. For the primary outcome, the ropivacaine plasma level, among- and within-groups differences for repeated measures were tested with a general linear model followed by adjustment according to the Sidak method. For secondary outcomes, frequency distributions were analyzed with a Chi-square test followed by a multiple regression approach using adjusted residuals and Bonferoni post hoc test, if appropriate. One-way ANOVA 
followed by Bonferroni adjustment or Kruskal Wallis test followed by Dunn-Bonferroni test for multiple comparison were used for independent parameters depending on the data distribution. Among- and within-groups differences for repeated measures were tested with a general linear model followed by adjustment according to the Sidak method. Since this study was planned as an explorative trial, no sample size estimation was performed. We opted for 30 patients (10 per group).

\section{Results}

Over a period of 7 months, 30 consecutive patients undergoing CEA were enrolled in the trial (Fig. 3). All patients completed the follow-up according to the trial protocol. The three groups were comparable regarding baseline characteristics (Table 2).

\section{Block execution and performance}

The identification of the nerve roots, of the intermediate cervical plexus, of the ICA and the bifurcation of carotid artery using ultrasound was successful in all patients. The distances from the skin to the ICA was $1.8 \pm 0.3 \mathrm{~cm}$ in the DCB group, $2.1 \pm 0.3 \mathrm{~cm}$ in the ICB group and $2.1 \pm 0.5 \mathrm{~cm}$ in the PVB group $(p=0.143)$. The time required to perform the block was significantly higher in the DCB group compared to PVB $(p=0.003$, Table 3$)$. The time until full expression of regional anesthesia is depicted in Fig. 4. All three groups showed sufficient analgesia in the dermatomes $\mathrm{C} 3$ and $\mathrm{C} 4$, but PVB was the only block providing analgesia in the dermatome $\mathrm{C} 2$ in all patients.

During surgery, 18 patients complained about pain NAS $\geq 2$ resulting in supplementation of the block with local lidocaine $1 \%$ by the surgeon ( 6 vs. 8 vs. 4 patients, DCB vs. ICB vs. PVB, $p=0.189$, respectively). In the PVB group a lower dosage of supplementation was required (Table 3). No conversion to general anesthesia due to an incomplete block or any other reasons was necessary. The duration of surgery was $103 \pm 33$ mins in the DCB group, $103 \pm 17$ mins in the ICB and $107 \pm 21$ min in the PVB group $(p=0.874)$. The cross-clamping time was $37 \pm 10 \mathrm{~min}$ in the DCB group, $37 \pm 9 \mathrm{~min}$ in the ICB group and $29 \pm 5$ min in group with PVB $(p=0.061)$. Planned or unplanned shunt placement was not necessary in any case. Surgeons' satisfaction was higher in the PVB group compared to ICB and patients' satisfaction was higher in the PVB group compared to ICB and TCB group (Fig. 5). Hemodynamic and functional data as well as cardiac biomarkers are shown in Additional file 1: Tables S1, S2 and Fig. S1. The length of hospital stay was $7.7 \pm 4.7$ days in the DCB group, $5.2 \pm 1.1$ days in the ICB group and $5.3 \pm 0.9$ days in the PVB group $(p=0.610)$. There were no in-hospital deaths observed.

\section{Ropivacaine plasma concentration}

The ropivacaine plasma concentration is shown in Fig. 6. The plasma concentration of ropivacaine was significantly higher in the DCB and PVB group compared to the ICB group (DCB vs. ICB, $p<0.001$; DCB vs. PVB, $p=0.001$; ICB vs. PVB, $p=0.008$; respectively). There was no adverse event related to the systemic plasma level of ropivacaine in all groups.

\section{Neurological complications}

No patient suffered from new intra- or postoperative central neurological deficits. Horner syndrome, hypoglossal

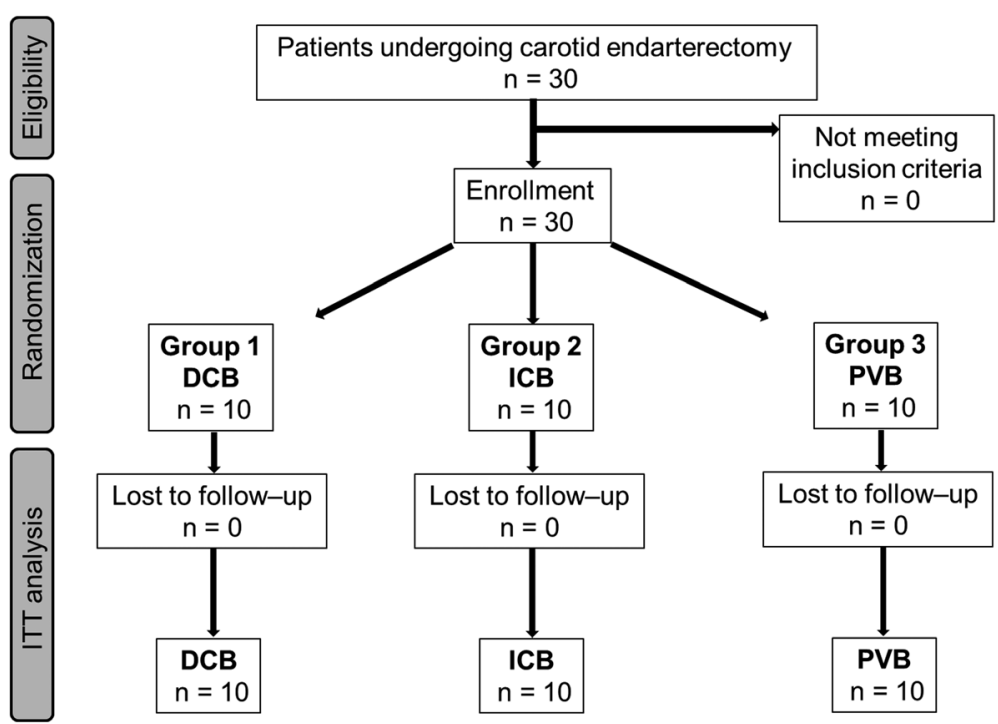

Fig. 3 Flow chart of enrolled patients. ITT: intention-to-treat analysis, DCB: deep cervical block, ICB: intermediate cervical block, PVB: intermediate cervical block with perivascular infiltration of the internal carotid artery 
Table 2 Baseline characteristics

\begin{tabular}{|c|c|c|c|}
\hline & $\begin{array}{l}\text { DCB } \\
(n=10)\end{array}$ & $\begin{array}{l}\text { ICB } \\
(n=10)\end{array}$ & $\begin{array}{l}\text { PVB } \\
(n=10)\end{array}$ \\
\hline Age [years] & $67 \pm 10$ & $70 \pm 9$ & $72 \pm 8$ \\
\hline Sex male & $7(70.0)$ & $9(90.0)$ & $8(80.0)$ \\
\hline female & $3(30.0)$ & $1(10.0)$ & $2(20.0)$ \\
\hline Bodyweight [kg] & $72 \pm 8$ & $83 \pm 8$ & $84 \pm 9$ \\
\hline Body height [cm] & $167 \pm 8$ & $174 \pm 8$ & $170 \pm 6$ \\
\hline BMI $\left[\mathrm{kg} / \mathrm{m}^{2}\right]$ & $25.9 \pm 1.6$ & $27.3 \pm 2.2$ & $29.1 \pm 3.4$ \\
\hline \multicolumn{4}{|l|}{ Stenosis } \\
\hline Grade [\%] & $81 \pm 7$ & $82 \pm 6$ & $82 \pm 9$ \\
\hline Sight left & $5(50.0)$ & $5(50.0)$ & $5(50.0)$ \\
\hline right & $5(50.0)$ & $5(50.0)$ & $5(50.0)$ \\
\hline asymptomatic & $6(60.0)$ & $6(60.0)$ & $7(70.0)$ \\
\hline symptomatic & $4(40.0)$ & $4(40.0)$ & $3(30.0)$ \\
\hline ASA $\|$ & $6(60.0)$ & $6(60.0)$ & $5(50.0)$ \\
\hline III & $4(40.0)$ & $4(40.0)$ & $5(50.0)$ \\
\hline \multicolumn{4}{|l|}{ Comorbidities } \\
\hline Arterial hypertension & $10(100.0)$ & $10(100.0)$ & $10(100.0)$ \\
\hline Diabetes mellitus & $4(40.0)$ & $4(40.0)$ & $5(50.0)$ \\
\hline Hyperlipoproteinema & $10(100.0)$ & $9(90.0)$ & $10(100.0)$ \\
\hline PAOD & $6(60.0)$ & $1(10.0)$ & $7(70.0)$ \\
\hline CAD & $4(40.0)$ & $4(40.0)$ & $5(50.0)$ \\
\hline $\mathrm{AMl}$ & $1(10.0)$ & $1(10.0)$ & $2(20.0)$ \\
\hline Stent & $2(20.0)$ & $2(20.0)$ & $2(20.0)$ \\
\hline CABG & $1(10.0)$ & $0(0.0)$ & $3(30.0)$ \\
\hline Artrial fibrillation & $1(10.0)$ & $0(0.0)$ & $0(0.0)$ \\
\hline COPD & $2(20.0)$ & $2(20.0)$ & $1(10.0)$ \\
\hline CKD & $3(30.0)$ & $2(20.0)$ & $1(10.0)$ \\
\hline Nicotine abuse & $7(70.0$ & $7(70.0$ & $3(30.0)$ \\
\hline Alcohol abuse & $5(50.0)$ & $3(30.0)$ & $0(0.0)$ \\
\hline
\end{tabular}

Values are given as mean \pm standard deviation or absolute number and percentage. $A M I$ acute myocardial infarction in medical history, ASA American Society of Anesthesiology physic status, $B M I$ body mass index, $C A D$ coronary artery disease, $C A B G$ coronary artery bypass graft, $C K D$ chronic kidney disease, $C O P D$ chronic obstructive pulmonary disease, $D C B$ deep cervical block, ICB intermediate cervical block, $P A O D$ peripheral artery occlusive disease, $P V B$ intermediate cervical block with perivascular infiltration of the internal carotid artery

nerve or permanent facial nerve paralysis were not observed in any patient. Hemi-diaphragmatic impairment caused by phrenic nerve paralysis was associated with a more frequent occurrence in the DCB group $(p=0.022$, Fig. 7). None of these patients with hemi-diaphragmatic paralysis showed signs of respiratory distress.

\section{Discussion}

\section{Major findings}

The major findings of the present study are:
1. The plasma level of local anesthetic was significantly higher in the DCB group and PVB group compared to ICB alone, without causing adverse events.

2. Impairment of ventilation due to hemidiaphragmatic paralysis was frequently observed in the DCB group.

3. The PVB is a feasible regional anesthetic technique providing sufficient analgesia for CEA in all of the desired dermatomes $\mathrm{C} 2-\mathrm{C} 4$.

This is the first trial comparing ropivacaine plasma levels with PVB, DCB and ICB. Regional anesthesia and CEA were performed by senior physicians. We tried to reduce bias by blinding the patients, the surgeons and outcome assessors to groups. In addition to improve adherence to blinding, the anesthesiologist performing the cervical block did not treat the patient during surgery. Adherence to allocation concealment and blinding of participants, study personnel and outcome assessors were maintained throughout the trial.

\section{Regional anesthesia in CEA}

The implementation of ultrasound in regional anesthesia has increased safety and efficacy by direct visualization of the target structure and the needle tip as well as by observation of local anesthetic spread during injection. Despite these advantages, even the ultrasound guided regional anesthetic techniques require a high level of local anesthetic infiltration by surgeons [14-16]. In our opinion, an important reason for the high rate of local anesthetic supplementation is the complex innervations of the neurovascular sheath by the vagal and glossopharyngeal nerve. In several previous studies, ultrasound guided perivascular infiltration of the ICA decreased the necessity of local anesthetic supplementation by surgeons and increased the efficacy of regional anesthesia $[8,17,18]$. However, the influence of perivascular infiltration on local anesthetic plasma concentration or the risk of phrenic nerve paralysis were not compared so far.

\section{Ropivacaine plasma levels}

The plasma concentration of local anesthetic depends on different conditions. Particularly, the type of local anesthetic as well as the vascularization of the puncture site are important factors of local anesthetic absorption. Ropivacaine is associated with low lipid solubility and provides a better neurological and cardiac toxicity profile than bupivacaine. Additionally, the vasoconstrictive effetcs of ropivacaine delays the absorption of the local anesthetic and may therefore be particularly suitable for regional anesthetic techniques in highly vascularized regions. Besides this advantages also for ropivacaine severe 
Table 3 Supplementation of block with additional local anesthesia by the surgeon and NAS results $(0=$ no pain $-10=$ worst imaginable pain)

\begin{tabular}{|c|c|c|c|c|c|}
\hline & & $\begin{array}{l}\text { DCB } \\
(n=10)\end{array}$ & $\begin{array}{l}\text { ICB } \\
(n=10)\end{array}$ & $\begin{array}{l}\text { PVB } \\
(n=10)\end{array}$ & $\begin{array}{l}P \\
\text { value }\end{array}$ \\
\hline Duration of block dispo & on $[\mathrm{min}]$ & $15.9 \pm 2.8$ & $11.5 \pm 2.1$ & $14.1 \pm 2.0$ & $0.019^{*}$ \\
\hline Additional lidocaine [No & & $6(60.0)$ & $8(80.0)$ & $4(40.0)$ & n.a. \\
\hline Additional lidocaine [ms & & $90.0(50.0,100.0,145.0,160.0)$ & $150.0(50.0,100.0,200.0,300.0)$ & $85.0(30.0,35.0,120.0,120.0)$ & n.a. \\
\hline Numeric analog scale* & RA & $2(0,2,4,5)$ & $3(0,1,3,5)$ & $2(1,1,2,3)$ & 0.336 \\
\hline & Incision & $1(0,1,3,5)$ & $2(0,1,2,5)$ & $1(0,1,2,3)$ & 0.659 \\
\hline & Retractor & $1(0,1,2,4)$ & $2(0,1,3,3)$ & $1(0,1,2,3)$ & 0.301 \\
\hline & Dissection & $2(1,1,4,6)$ & $1(0,3,4,5)$ & $2(1,2,2,3)$ & 0.061 \\
\hline & Clamping & $2(0,1,2,2)$ & $2(0,2,3,3)$ & $2(1,2,3,5)$ & 0.153 \\
\hline & Suture & $1(0,1,1,5)$ & $2(0,1,2,3)$ & $1(0,1,2,2)$ & 0.189 \\
\hline
\end{tabular}

Values are given as mean \pm standard deviation, median (minimum, $25 \%$ percentile, $75 \%$ percentile, maximum) or absolute number (percentage) as appropriate. Differences among groups were tested with Kruskal-Wallis followed by Dunn-Bonferroni test. Statistical significance was considered to be at two-sided $p<0.05$. $D C B$ deep cervical block, ICB intermediate cervical block, $P V B$ intermediate cervical block with perivascular infiltration of the internal carotid artery, $R A$ regional anesthesia, No number, $n . a$ statistics not applicable due to low patient number in the PVB group. *: $p<0.001$ DCB vs. ICB
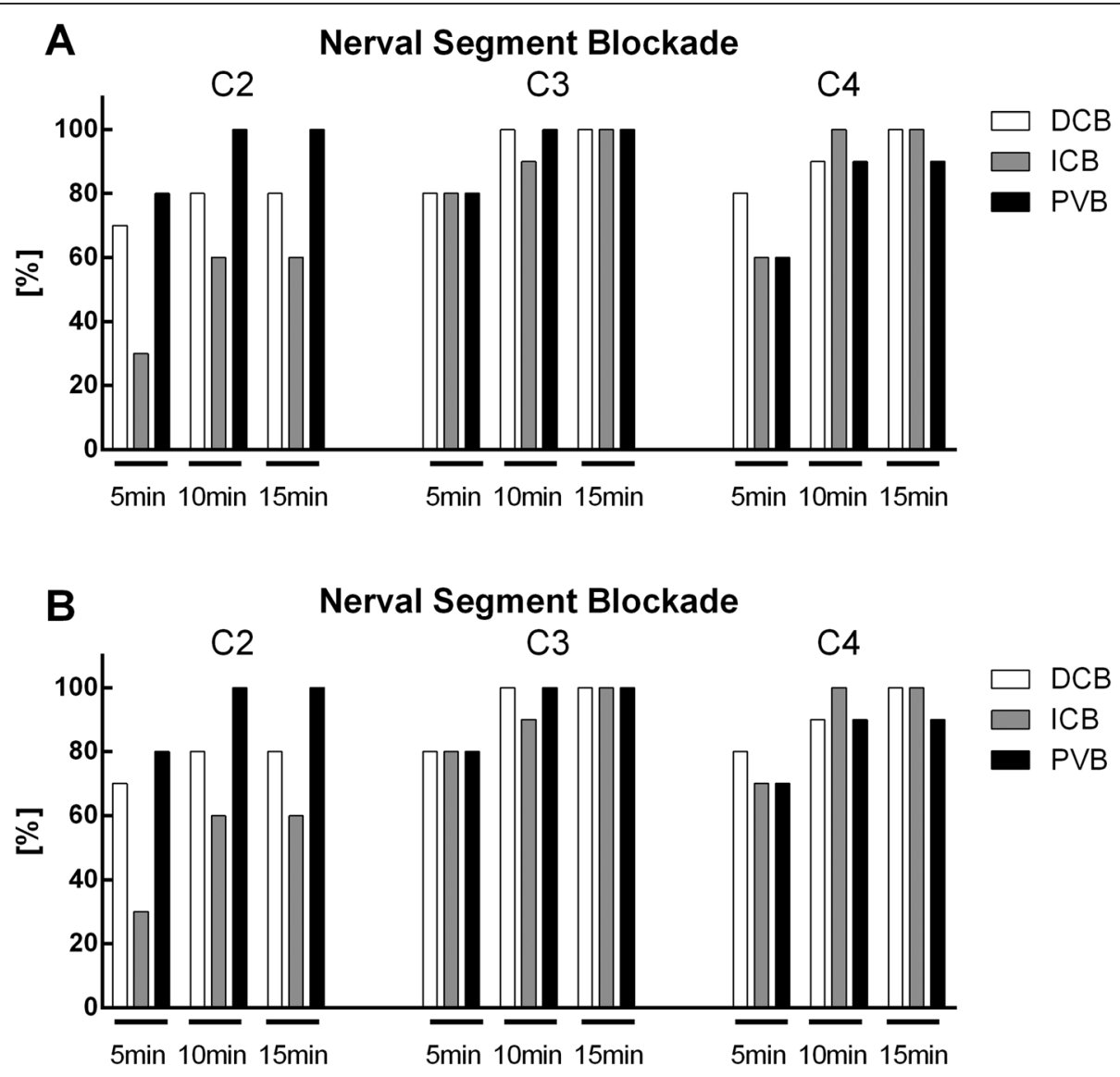

Fig. 4 Block distention in the dermatomes C2 to C4. Block distention was determined using peaked/blunt discrimination (a) or warm/cold discrimination (b). Values are given as percentage and were measured $5 \mathrm{~min}, 10 \mathrm{~min}$ and $15 \mathrm{~min}$ after completion of block placement, respectively. DCB: deep cervical block, ICB: intermediate cervical block, PVB: intermediate cervical block with perivascular infiltration of the internal carotid artery 


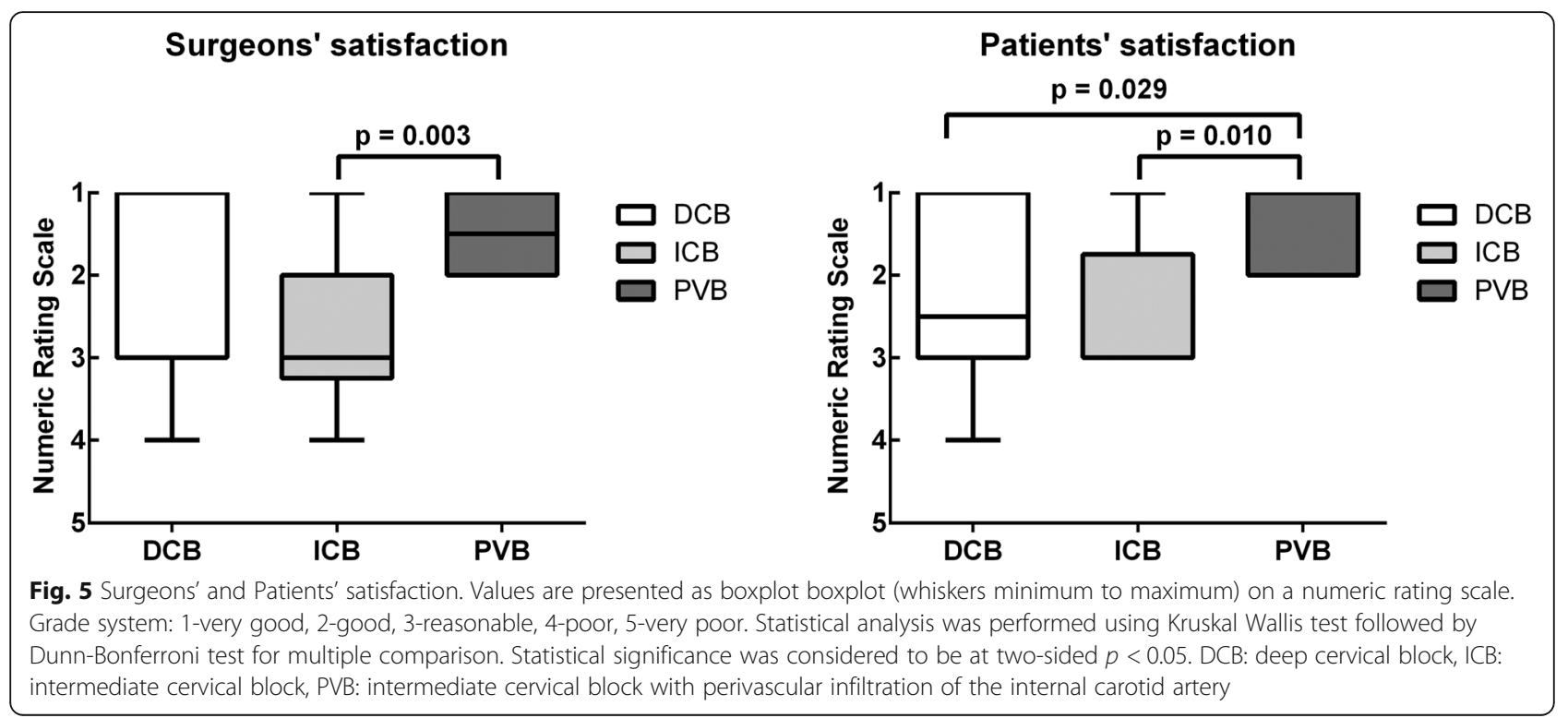

complications up to local anesthetic intoxications with cardiac arrest are reported [19, 20].

In carotid endarterectomy cerebral seizure by local anesthetic intoxication can lead to the inability to properly monitor neurological symptoms and increases the oxygen consumption of the brain. Davies et al. reported two cases of local anesthetic intoxications in 1000 carotid endarterectomies, which equals an incidence of $0.2 \%$ [5]. The occurrence of cerebral symptoms depends on the maximum ropivacaine plasma concentration as well as the slope of the plasma level increase.






\section{Phrenic Nerve Paresis}

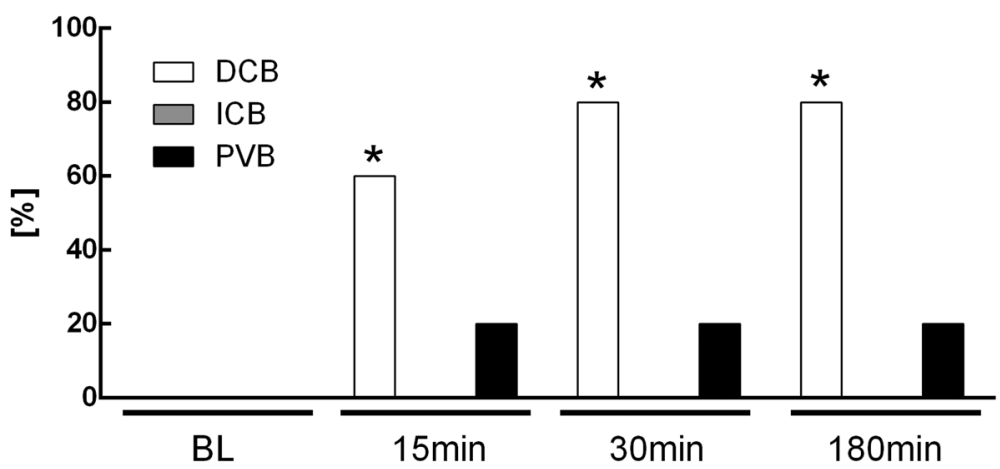

Fig. 7 Incidence of phrenic nerve paralysis. Values are given as percentage at baseline (BL), $5 \mathrm{~min}, 30 \mathrm{~min}$, and $180 \mathrm{~min}$ after completion of block placement, respectively. A Chi-square test with multiple regression approach and Bonferroni post hoc test were performed. Statistical significance was accepted a $p<0.05$. DCB: deep cervical block, ICB: intermediate cervical block, PVB: intermediate cervical block with perivascular infiltration of the internal carotid artery. ${ }^{*}: p<0.05$

In the present study, the lowest peak concentrations of ropivacaine $(0.3 \mu \mathrm{g} / \mathrm{mL})$ were measured in the intermediate cervical block group and the highest peak concentrations $(2.1 \mu \mathrm{g} / \mathrm{mL})$ in the deep cervical block group. However, the detected ropivacaine plasma concentrations were well below the threshold for early neurological toxicity symptoms to be $2.2 \mu \mathrm{g} / \mathrm{mL}$ as described by Knudsen et al. [21]. Different groups reported comparable plasma concentrations of ropivacaine after interscalene or deep cervical plexus block [22]. In contrast, few studies examined the ropivacaine plasma concentrations after intermediate cervical block. Koköfer et al. reported plasma concentrations after ultrasound guided triple injection technique (intermediate cervical block, perivascular infiltration and subcutane infiltration) [7]. This group used $20 \mathrm{~mL}$ ropivacaine $0.375 \%$ or $0.75 \%$ for intermediate cervical block and prilocaine $1 \%$ for the perivascular infiltration of ICA. Additionally, prilocaine $1 \%$ was used also for the subcutaneous infiltration along the anterior border of sternocleidomastoid muscle. The peak plasma levels in the ropivacaine $0.375 \%$ group ranged from 4 to $7 \mu \mathrm{g} / \mathrm{mL}$ and in the ropivacaine $0.75 \%$ group from 5 to $10 \mu \mathrm{g} / \mathrm{mL}$. In contrast, the peak plasma levels for intermediate cervical block in our study ranged from 0.3 to $0.6 \mu \mathrm{g} / \mathrm{mL}$. Several reasons may explain these different results of local anesthetic plasma levels after intermediate cervical plexus block. The use of two different local anesthetics by Koköfer could have led to an increase of ropivacaine plasma concentration. Another cause might be the binding of ropivacaine to $\alpha_{1}$-acid glycoprotein, which may markedly affect the pharmacokinetics of ropivacaine [23]. However, $\alpha_{1}$-acid glycoprotein levels were measured neither in the present study, nor by Koköfer [7].

The effects of perivascular infiltration on ropivacaine plasma concentration was not examined in any study.
Although Koköfer et al. performed a perivascular infiltration, the effect on plasma concentration was not assessed. He used prilocaine $1 \%$ for perivascular infiltration as well as for subcutaneous infiltration. In our study, we applied only ropivacaine for all regional anesthetic techniques. The ropivacaine plasma concentrations of the perivascular group were significantly higher than for intermediate block alone. In our opinion, the reason of the higher ropivacaine concentration is the larger volume of local anesthetic applied in the perivascular group compared to the intermediate block alone suggesting similar tissue adsorption characteristics.

The threshold plasma concentration at which central nerve system toxicity occurs may be related more to the rate of increase of the serum concentration rather than to the total amount of drug injected. Wulf and colleagues examined the plasma concentration after combined ilioinguinal-iliohypogastric block with ropivacaine [24]. The peak plasma concentrations of ropivacaine were $1.5 \mu \mathrm{g} / \mathrm{mL}$ and occurred $45 \mathrm{~min}$ after injection. In contrast, Rettig et al. examined the plasma concentrations of ropivacaine after brachial plexus blockade using four different approaches [22]. The authors reported the lateral and posterior interscalene block to be associated with earlier (10 and $15 \mathrm{~min}$, respectively) and high peak plasma concentrations of local anesthetic $(4,4 \mu \mathrm{g} / \mathrm{mL}$ and $4,5 \mu \mathrm{g} / \mathrm{mL}$ ). According to these studies, the increase in plasma concentrations is significantly influenced by the anatomic region of regional anesthesia. In the present study, the fastest increase in ropivacaine concentration was observed in the deep cervical block group. However, no cerebral signs of local anesthetic intoxication were observed. The time to reach the maximum concentration was 5 to $10 \mathrm{~min}$. Similar results were reported by Merle, who found times of 5 to $17 \mathrm{~min}$ for the classic deep cervical block [25]. In contrast to deep 
cervical block, in our study the increase in ropivacaine plasma concentrations was significantly slower (10 to 20 min) for intermediate cervical plexus block alone. Similar results were observed for combination of intermediate cervical block and perivascular infiltration. In this group the time to reach the maximum concentration was 10 to $20 \mathrm{~min}$. In our opinion, the reason of the faster increase in ropivacaine plasma concentration is a pronounced vascularization in the deep cervical space.

\section{Phrenic nerve paralysis}

Phrenic nerve paralysis can occur during cervical block due to the close anatomical relation. The phrenic nerve origins mainly from the $\mathrm{C} 4$ root, with variable portions from the C3 and C5 root [26]. After formation of the phrenic nerve at the upper lateral border of the anterior scalene muscle the nerve continues caudally between the ventral surface of the anterior scalene muscle and prevertebral fascial layer that covers this muscle and is therefore separated from the brachial plexus only by a thin fascial layer [26]. During regional anesthesia, a perioperative phrenic nerve paralysis can have various causes $[27,28]$. Temporary phrenic nerve palsies are most common after cardiac surgery but may also be caused by CEA due to traction or compression as well as local anesthetic supplementation [29].

In the current trial, all patients showed bilateral ventilation before regional anesthesia. In ten patients (DCB: $n=8$, PVB: $n=2$ ) a phrenic nerve paresis was observed. None of these patients suffered from respiratory distress. The high rate of phrenic nerve paralysis in our investigation is not surprising for deep cervical block, where a phrenic nerve paralysis occur in 55 to $61 \%$ of the cases $[30,31]$. An even higher rate up to $100 \%$ of hemidiaphragmatic paralysis is reported by Urmey et al. for the interscalene brachial plexus block [32, 33]. Despite this high incidence of paralysis of the phrenic nerve, reports of significant shortness of breath or impairment of gas exchange are rare [33-35]. The occurrence of phrenic nerve paresis in the perivascular group is more difficult to explain. The precise anatomy of the deeper neck compartments is complex and has not been completely understood so far. For decades, the concept of impenetrability of the deep fascia of the neck for local anesthetics was indefeasible [36], but has been questioned recently $[37,38]$. These doubts are supported by case reports observing complications such as Horner syndrome after superficial blocks [39]. Furthermore, Pandit et al. described in a cadaver study penetration of a superficial injection of methylene blue to the nerve roots in the deep space [40]. Contrary, in another corpse study, Seidel and colleagues observed no spread of methylene blue through the deep cervical fascia [36]. Nevertheless, there were clear methodological differences between these two cadaver studies, especially in the fluid volume administered [36, 40]. In our opinion, larger volumes of local anesthetic may cause higher intracompartment pressures and therefore enhance a deeper spread of the local anesthetic via the anatomic pathways described by Pandit $[38,40]$. This may result in phrenic nerve paresis in the deep cervical compartment. However, further detailed studies are warranted to prove this hypothesis.

\section{Limitations}

The present trial has several limitations. First, the present trial was an explorative pilot study. Therefore, no sample size calculation was performed. Second, the hemi-diaphragmatic paresis was diagnosed indirectly through decrease of regional ventilation in one lung via EIT. This functional approach to phrenic nerve paresis was described by Reske and colleagues for interscalene brachial plexus block in a small patient collective [13]. The EIT has the major advantage that the impairment of ventilation could easily be detected at the bedside [41]. Ultrasound imaging of the diaphragm is more observer dependent and can be difficult in obese patients. In contrast, the EIT has also been used in obese patients by Nestler et al. [42]. However, the EIT method for detecting hemi-diaphragmatic paresis has not been validated in a larger patient collective so far. Parallel ultrasound imaging of the diaphragm was not performed in the current trial. Third, the assessment of patient and surgeon satisfaction with the respective block was subjective. The simple grading scale for satisfaction from 1 to 5 was chosen for patients' feasibility. The same grading was used for surgeons' rating with regards to comparability. Fourth, the individual patient pain and convenience level in the operating room may have influenced the surgeons' decision for additional administration of local anesthetic in the operating situs.

\section{Implications for further studies}

Future trials investigating the effects of different regional anesthetic techniques such as DCB, ICB and PVB on patient safety, systemic local anesthetic concentration and side effects are warranted. Such a trial should be prospective, randomized, controlled and ideally triple blind focusing for instance on postoperative pulmonary complications caused by phrenic nerve paralysis with dual assessment of diaphragm function by EIT and ultrasound as primary outcome. The present trial may provide a basis for sample size calculation. However, the evaluation of systemic toxic side effects of local anesthetics during regional anesthesia for CEA will be difficult due to the rare occurrence [5]. A prospective observational trial focusing on the occurrence of seizures and new arrhythmias in context to the regional 
anesthetic technique may help to further investigate these systemic and clinical relevant side effects. The adaptation of the data entry in national or international databases for CEA regarding the specific regional anesthetic technique and type of anesthetic may help researchers to get access to a larger data set for a retrospective trial.

\section{Conclusions}

The ultrasound guided intermediate cervical block with perivascular infiltration of the internal carotid artery is a safe and feasible technique for carotid endarterectomy. However, further studies in a larger patient collective are warranted to evaluate rare side effects related to the area of administration and systemic plasma concentration of the local anesthetic.

\section{Supplementary information}

Supplementary information accompanies this paper at https://doi.org/10 1186/s12871-019-0890-8.

Additional file 1: Figure S1. Cardiac biomarkers. Table S1.

Hemodynamic data and Table S2. Blood gas analysis data.

\begin{abstract}
Abbreviations
CEA: Carotid endarterectomy; CONSORT: Consolidated Standards of Reporting Trials; DCB: Deep cervical block; EIT: Electrical impedance tomography; ICA: Internal carotid artery; ICB: Intermediate cervical block; PVB: Intermediate cervical block with perivascular infiltration of the internal carotid artery; ROI: Region of interest
\end{abstract}

\section{Acknowledgements}

The authors thank the anesthetic and surgical staff of the Department of Visceral, Thoracic and Vascular Surgery, University Hospital Carl Gustav Carus, Technische Universität Dresden, Dresden, Germany. The authors express their special gratitude to Rüdiger Paul, who helped performing regional anesthesia and to Dr. Anja Braune, who developed the Matlab routine for EIT analysis.

\section{Authors' contributions}

[TR]1 and CU contributed equally to this manuscript. [TR]1 designed the study protocol, collected data and contributed to manuscript preparation. CU helped with data collection, performed the statistical data analysis and helped preparing the manuscript. JP performed the ropivacaine plasma level measurements and contributed to the manuscript preparation. SL performed carotid surgery and helped preparing the manuscript. [TR]2: helped preparing the manuscript. TK helped preparing the manuscript. PMS helped preparing the manuscript and interpreting the data. SK contributed to manuscript preparation and interpretation of the data. All authors have read and approved the manuscript.

\section{Funding}

We acknowledge support by the Open Access Publication Funds of the SLUB/TU Dresden.

\section{Availability of data and materials}

The datasets used and/or analyzed during the current study are available from the corresponding author on request.

\section{Ethics approval and consent to participate}

The present trial was approved by the local institutional review board of the TU Dresden, the "Ethikkomission an der Technischen Universität Dresden", Dresden, Germany (approval number: EK 130042013). Written informed consent was obtained from all participants with the informed consent form prior to trial enrollment.

\section{Consent for publication}

Not Applicable.

\section{Competing interests}

The authors declare that they have no competing interests.

\section{Author details}

${ }^{1}$ Department of Anaesthesiology and Critical Care Medicine, University Hospital Carl Gustav Carus Dresden, Technische Universität Dresden, Fetscherstr. 74, 01307 Dresden, Germany. Institute of Legal Medicine, Technische Universität Dresden, Dresden, Germany. ${ }^{3}$ Department of Visceral, Thoracic and Vascular Surgery, University Hospital Carl Gustav Carus, Technische Universität Dresden, Dresden, Germany. ${ }^{4}$ Department of General Surgery, University Hospital of Friedrich-Alexander-University, Erlangen, Germany.

Received: 11 April 2019 Accepted: 12 November 2019

Published online: 26 November 2019

\section{References}

1. Sbarigia E, Schioppa A, Misuraca M, Panico MA, Battocchio C, Maraglino C, Speziale F, Fiorani P. Somatosensory evoked potentials versus locoregional anaesthesia in the monitoring of cerebral function during carotid artery surgery: preliminary results of a prospective study. Eur J Vasc Endovasc Surg. 2001;21(5):413-6.

2. Group GTC, Lewis SC, Warlow CP, Bodenham AR, Colam B, Rothwell PM, Torgerson D, Dellagrammaticas D, Horrocks M, Liapis C, et al. General anaesthesia versus local anaesthesia for carotid surgery (GALA): a multicentre, randomised controlled trial. Lancet. 2008;372(9656):2132-42.

3. Stoneham MD, Doyle AR, Knighton JD, Dorje P, Stanley JC. Prospective, randomized comparison of deep or superficial cervical plexus block for carotid endarterectomy surgery. Anesthesiology. 1998;89(4):907-12.

4. Pandit JJ, Satya-Krishna R, Gration P. Superficial or deep cervical plexus block for carotid endarterectomy: a systematic review of complications. Br J Anaesth. 2007;99(2):159-69.

5. Davies MJ, Silbert BS, Scott DA, Cook RJ, Mooney PH, Blyth C. Superficial and deep cervical plexus block for carotid artery surgery: a prospective study of 1000 blocks. Reg Anesth. 1997;22(5):442-6.

6. Roessel T, Wiessner D, Heller AR, Zimmermann T, Koch T, Litz RJ. Highresolution ultrasound-guided high interscalene plexus block for carotid endarterectomy. Reg Anesth Pain Med. 2007:32(3):247-53.

7. Kokofer A, Nawratil J, Felder TK, Stundner O, Mader N, Gerner P. Ropivacaine $0.375 \%$ vs. $0.75 \%$ with prilocaine for intermediate cervical plexus block for carotid endarterectomy: a randomised trial. Eur J Anaesthesiol. 2015;32(11): 781-9.

8. Rossel T, Kersting S, Heller AR, Koch T. Combination of high-resolution ultrasound-guided perivascular regional anesthesia of the internal carotid artery and intermediate cervical plexus block for carotid surgery. Ultrasound Med Biol. 2013:39(6):981-6.

9. Karmakar MK, Kwok WH, Ho AM, Tsang K, Chui PT, Gin T. Ultrasound-guided sciatic nerve block: description of a new approach at the subgluteal space. Br J Anaesth. 2007:98(3):390-5.

10. Guntz E, Herman P, Debizet E, Delhaye D, Coulic V, Sosnowski M. Sciatic nerve block in the popliteal fossa: description of a new medial approach. Can J Anaesth. 2004;51(8):817-20.

11. Greher M, Scharbert G, Kamolz LP, Beck H, Gustorff B, Kirchmair L, Kapral S. Ultrasound-guided lumbar facet nerve block: a sonoanatomic study of a new methodologic approach. Anesthesiology. 2004;100(5):1242-8.

12. Eldridge SM, Chan CL, Campbell MJ, Bond CM, Hopewell S, Thabane L, Lancaster GA, Group PC. CONSORT 2010 statement: extension to randomised pilot and feasibility trials. BMJ. 2016;355:15239.

13. Wiegel M, Hammermuller $S$, Wrigge $H$, Reske AW. Electrical impedance tomography visualizes impaired ventilation due to Hemidiaphragmatic paresis after Interscalene brachial plexus block. Anesthesiology. 2016; 125(4):807.

14. Barone M, Diemunsch P, Baldassarre E, Oben WE, Ciarlo M, Wolter J, Albani A. Carotid endarterectomy with intermediate cervical plexus block. Tex Heart Inst J. 2010;37(3):297-300. 
15. Alilet A, Petit P, Devaux B, Joly C, Samain E, Pili-Floury S, Besch G. Ultrasound-guided intermediate cervical block versus superficial cervical block for carotid artery endarterectomy: the randomized-controlled CERVECHO trial. Anaesth Crit Care Pain Med. 2017;36(2):91-5.

16. Calderon AL, Zetlaoui P, Benatir F, Davidson J, Desebbe O, Rahali N, Truc C, Feugier P, Lermusiaux P, Allaouchiche B, et al. Ultrasound-guided intermediate cervical plexus block for carotid endarterectomy using a new anterior approach: a two-Centre prospective observational study. Anaesthesia. 2015;70(4):445-51.

17. Hoefer J, Pierer E, Rantner B, Stadlbauer KH, Fraedrich G, Fritz J, Kleinsasser A, Velik-Salchner C. Ultrasound-guided regional anesthesia for carotid endarterectomy induces early hemodynamic and stress hormone changes. J Vasc Surg. 2015;62(1):57-67.

18. Madro P, Dabrowska A, Jarecki J, Garba P. Anaesthesia for carotid endarterectomy. Ultrasound-guided superficial/intermediate cervical plexus block combined with carotid sheath infiltration. Anaesthesiol Intensive Ther. 2016:48(4):234-8.

19. Sindjelic RP, Vlajkovic GP, Lucic M, Koncar I, Kostic D, Davidovic LB. Incidence of and indications for conversion of cervical plexus block to general anesthesia in patients undergoing carotid surgery: a single center experience. J Cardiovasc Surg. 2015;56(3):441-6.

20. Litz RJ, Popp M, Stehr SN, Koch T. Successful resuscitation of a patient with ropivacaine-induced asystole after axillary plexus block using lipid infusion. Anaesthesia. 2006;61(8):800-1.

21. Knudsen K, Beckman Suurkula M, Blomberg S, Sjovall J, Edvardsson N. Central nervous and cardiovascular effects of i.v. infusions of ropivacaine, bupivacaine and placebo in volunteers. Br J Anaesth. 1997;78(5):507-14.

22. Rettig HC, Lerou JG, Gielen MJ, Boersma E, Burm AG. The pharmacokinetics of ropivacaine after four different techniques of brachial plexus blockade. Anaesthesia. 2007;62(10):1008-14

23. Simpson D, Curran MP, Oldfield V, Keating GM. Ropivacaine: a review of its use in regional anaesthesia and acute pain management. Drugs. 2005; 65(18):2675-717.

24. Wulf $\mathrm{H}$, Worthmann $\mathrm{F}$, Behnke $\mathrm{H}$, Bohle AS. Pharmacokinetics and pharmacodynamics of ropivacaine $2 \mathrm{mg} / \mathrm{mL}, 5 \mathrm{mg} / \mathrm{mL}$, or $7.5 \mathrm{mg} / \mathrm{mL}$ after ilioinguinal blockade for inguinal hernia repair in adults. Anesth Analg. 1999; 89(6):1471-4.

25. Merle JC, Mazoit JX, Desgranges P, Abhay K, Rezaiguia S, Dhonneur G, Duvaldestin P. A comparison of two techniques for cervical plexus blockade: evaluation of efficacy and systemic toxicity. Anesth Analg. 1999; 89(6):1366-70

26. Riazi S, Carmichael N, Awad I, Holtby RM, McCartney CJ. Effect of local anaesthetic volume (20 vs $5 \mathrm{ml}$ ) on the efficacy and respiratory consequences of ultrasound-guided interscalene brachial plexus block. Br J Anaesth. 2008;101(4):549-56.

27. Wakeno M, Sakamoto S, Asai T, Hirose T, Shingu K. A case of diaphragmatic paralysis in a patient with diabetes mellitus after surgery in prolonged prone position. Masui. 2001;50(9):1019-21.

28. Schram DJ, Vosik W, Cantral D. Diaphragmatic paralysis following cervical chiropractic manipulation: case report and review. Chest. 2001;119(2): 638-40.

29. Kunisawa T, Hanada S, Takeuchi S, Iwasaki H. Transient phrenic nerve paralysis after carotid endarterectomy in a patient with asthma. J Anesth. 2010;24(5):817-8.

30. Emery G, Handley G, Davies MJ, Mooney PH. Incidence of phrenic nerve block and hypercapnia in patients undergoing carotid endarterectomy under cervical plexus block. Anaesth Intensive Care. 1998;26(4):377-81.

31. Castresana MR, Masters RD, Castresana EJ, Stefansson S, Shaker IJ, Newman $\mathrm{WH}$. Incidence and clinical significance of hemidiaphragmatic paresis in patients undergoing carotid endarterectomy during cervical plexus block anesthesia. J Neurosurg Anesthesiol. 1994;6(1):21-3.

32. Urmey WF, Talts KH, Sharrock NE. One hundred percent incidence of hemidiaphragmatic paresis associated with interscalene brachial plexus anesthesia as diagnosed by ultrasonography. Anesth Analg. 1991;72(4): 498-503.

33. Urmey WF, McDonald M. Hemidiaphragmatic paresis during interscalene brachial plexus block: effects on pulmonary function and chest wall mechanics. Anesth Analg. 1992;74(3):352-7.

34. Mian A, Chaudhry I, Huang R, Rizk E, Tubbs RS, Loukas M. Brachial plexus anesthesia: a review of the relevant anatomy, complications, and anatomical variations. Clin Anat. 2014;27(2):210-21.
35. Knoblanche GE. The incidence and aetiology of phrenic nerve blockade associated with supraclavicular brachial plexus block. Anaesth Intensive Care. 1979;7(4):346-9.

36. Seidel R, Schulze M, Zukowski K, Wree A. Ultrasound-guided intermediate cervical plexus block. Anatomical study. Anaesthesist. 2015;64(6):446-50.

37. Nash L, Nicholson HD, Zhang M. Does the investing layer of the deep cervical fascia exist? Anesthesiology. 2005;103(5):962-8.

38. Pandit JJ, Dorje P, Satya-Krishna R. Investing layer of the cervical fascia of the neck may not exist. Anesthesiology. 2006;104(6):1344 author reply 1344-1345.

39. Flores S, Riguzzi C, Herring AA, Nagdev A. Horner's syndrome after superficial cervical plexus block. West J Emerg Med. 2015;16(3):428-31.

40. Pandit JJ, Dutta D, Morris JF. Spread of injectate with superficial cervical plexus block in humans: an anatomical study. Br J Anaesth. 2003;91(5):733-5.

41. Costa EL, Lima RG, Amato MB. Electrical impedance tomography. Curr Opin Crit Care. 2009;15(1):18-24.

42. Nestler C, Simon P, Petroff D, Hammermuller S, Kamrath D, Wolf S, Dietrich A, Camilo LM, Beda A, Carvalho AR, et al. Individualized positive endexpiratory pressure in obese patients during general anaesthesia: a randomized controlled clinical trial using electrical impedance tomography. Br J Anaesth. 2017;119(6):1194-205.

\section{Publisher's Note}

Springer Nature remains neutral with regard to jurisdictional claims in published maps and institutional affiliations.
Ready to submit your research? Choose BMC and benefit from:

- fast, convenient online submission

- thorough peer review by experienced researchers in your field

- rapid publication on acceptance

- support for research data, including large and complex data types

- gold Open Access which fosters wider collaboration and increased citations

- maximum visibility for your research: over $100 \mathrm{M}$ website views per year

At BMC, research is always in progress.

Learn more biomedcentral.com/submissions 